\title{
Survival benefit of bursectomy in patients with resectable gastric cancer: interim analysis results of a randomized controlled trial
}

\author{
Junya Fujita $\cdot$ Yukinori Kurokawa $\cdot$ Tomoyuki Sugimoto $\cdot$ Isao Miyashiro $\cdot$ \\ Shohei Iijima Y Yutaka Kimura -Shuji Takiguchi $\cdot$ Yoshiyuki Fujiwara • \\ Masaki Mori $\cdot$ Yuichiro Doki
}

Received: 7 February 2011/Accepted: 18 April 2011/Published online: 15 May 2011

(C) The International Gastric Cancer Association and The Japanese Gastric Cancer Association 2011

\begin{abstract}
Background Bursectomy is regarded as a standard surgical procedure during gastrectomy for serosa-positive gastric cancer in Japan. There is little evidence, however, that bursectomy has clinical benefit. We conducted a randomized controlled trial to demonstrate non-inferiority of treatment with the omission of bursectomy.

Methods Between July 2002 and January 2007, 210 patients with cT2-T3 gastric adenocarcinoma were intraoperatively randomized to radical gastrectomy and D2 lymphadenectomy with or without bursectomy. The primary endpoint was overall survival (OS). Secondary endpoints were recurrence-free survival, operative morbidity, and levels of amylase in drainage fluid on postoperative
\end{abstract}

\section{J. Fujita}

Department of Surgery, Toyonaka Municipal Hospital,

Toyonaka, Japan

Y. Kurokawa ( $₫) \cdot$ S. Takiguchi · Y. Fujiwara · M. Mori ·

Y. Doki

Department of Gastroenterological Surgery, Osaka University

Graduate School of Medicine, Osaka, Japan

e-mail: ykurokawa@gesurg.med.osaka-u.ac.jp

T. Sugimoto

Department of Biomedical Statistics, Osaka University Graduate

School of Medicine, Osaka, Japan

I. Miyashiro

Department of Surgery, Osaka Medical Center for Cancer

and Cardiovascular Disease, Osaka, Japan

S. Iijima

Department of Surgery, Minoh City Hospital, Minoh, Japan

Y. Kimura

Department of Surgery, NTT West Osaka Hospital, Osaka, Japan day 1 . Two interim analyses were performed, in September 2008 and August 2010.

Results Overall morbidity (14.3\%) and mortality (0.95\%) rates were the same in the two groups. The median levels of amylase in drainage fluid on postoperative day 1 were similar in the two groups $(P=0.543)$. In the second interim analysis, the 3 -year OS rates were $85.6 \%$ in the bursectomy group and $79.6 \%$ in the non-bursectomy group. The hazard ratio for death without bursectomy was 1.44 (95\% confidence interval $[\mathrm{CI}] 0.79-2.61 ; P=0.443$ for non-inferiority). Among 48 serosa-positive (pT3-T4) patients, the 3-year OS was $69.8 \%$ for the bursectomy group and $50.2 \%$ for the non-bursectomy group, conferring a hazard ratio for death of 2.16 (95\% CI $0.89-5.22$; $P=0.791$ for non-inferiority). More patients in the nonbursectomy group had peritoneal recurrences than in the bursectomy group (13.2 vs. $8.7 \%$ ).

Conclusions The interim analyses suggest that bursectomy may improve survival and should not be abandoned as a futile procedure until more definitive data can be obtained.

Keywords Omental bursectomy · Bursa omentalis . Interim analysis · Gastric cancer

\section{Introduction}

To accomplish the cure of gastric cancer by surgical treatment it is of prime importance to eliminate all cancer cells from the patient. Total resection of the bursa omentalis has developed as an essential part of radical gastrectomy with extended lymphadenectomy as treatment for advanced gastric cancer in Japan [1, 2]. The operative procedure of bursectomy includes removal of the anterior 
membrane of the transverse mesocolon as well as the pancreatic capsule after total omentectomy. The rationale for this procedure is that en-bloc resection of the postgastric cavity lining, which includes free cancer cells or micrometastases, may reduce the incidence of cancer recurrence [3-5]. According to the Japanese Gastric Cancer Association Gastric cancer treatment guidelines, bursectomy is recommended for tumors with invasion of the serosa [6]. In the past few decades, most Japanese surgeons have continued to perform D2 lymphadenectomy with bursectomy as the conventional operation for advanced gastric cancer.

It is apparent, however, that removing the mesocolon and pancreatic capsule is physically detrimental to patients and increases the risk of intraoperative and/or postoperative complications. Some researchers have remained skeptical about prophylactic bursectomy [7-9], as no prospective clinical trial has clarified the benefits or effectiveness of this surgical procedure.

We have conducted a prospective randomized controlled trial with a non-inferiority design to evaluate prophylactic bursectomy for gastric cancer patients. Previously we reported short-term results for the study, which detailed that experienced surgeons could safely perform bursectomy without increasing major surgical complications [10]. Here, we provide a preliminary report on the results of the first and second interim analyses.

\section{Methods}

\section{Patients}

Eligibility criteria for the study included: (1) histologically proven primary adenocarcinoma of the stomach, (2) a preoperative and intraoperative classification of $\mathrm{T} 2 \mathrm{~N} 0$, T3N0, T2N1, or T3N1 according to the Japanese classification of gastric carcinoma, second English edition [11], (3) a lack of non-curative surgical factors except for positive lavage cytology, (4) no Borrmann type 4 (linitis plastica) cases, (5) no prior chemotherapy or radiation therapy, (6) age 20-80 years with a performance status of 0-2 according to the Eastern Cooperative Oncology Group (ECOG) scale, (7) no history of gastrectomy or other malignancy during the previous 5 years. All patients gave written informed consent before undergoing randomization. The surgeon confirmed the eligibility criteria during surgery and phoned the data center to receive a randomly generated assignment. Patients were then randomized to either the bursectomy group (a D2 gastrectomy with bursectomy) or the non-bursectomy group (a D2 gastrectomy without bursectomy), using the minimization method, according to gender, clinical $\mathrm{T}$ stage (cT2 vs. cT3), and gastrectomy (total vs. distal subtotal gastrectomy). The study protocol was approved by the institutional review board at each of the participating hospitals.

\section{Surgery}

The surgeons performed a total or distal subtotal gastrectomy and a D2 lymph node dissection as standard treatment for advanced gastric cancers in both groups. In total gastrectomy for $\mathrm{T} 2$ or deeper tumor in the proximal third of the stomach, the spleen was removed, in principle, for splenic hilar lymphadenectomy. Pancreatectomy was confined to those patients whose pancreas was involved by tumor. The type of reconstruction and the indication for prophylactic cholecystectomy were not specified in the protocol.

The details of the surgical procedure for bursectomy were described previously [10]. In brief, in the bursectomy group, the peritoneal lining of the bursa omentalis was removed en bloc as much as possible from the anterior plane of the transverse mesocolon and the pancreas. As complete removal of the left side of the bursa omentalis did not allow for a distal subtotal gastrectomy, the pancreatic serosa was removed up to the proximal half of the splenic artery. For the transverse colon mesentery, the peritoneum was removed up to the left gastroepiploic artery. In the non-bursectomy group, only a minimal amount of peritoneum could be removed for lymph node dissection. An omentectomy was performed for both groups in this study.

Patients were enrolled from 11 hospitals belonging to the Osaka University Clinical Research Group for Gastroenterological Surgery. More than 50 gastrectomies were performed every year in these 11 hospitals. All operations were performed or supervised by senior surgeons who were members of the Japanese Gastric Cancer Association. During the planning of the study, all participating surgeons reached an agreement concerning the technical details of bursectomy.

\section{Endpoint evaluations}

The primary endpoint was overall survival (OS), defined as the time from randomization to death. Secondary endpoints were recurrence-free survival (RFS), operative morbidity, and levels of amylase in drainage fluid on postoperative day 1. RFS was defined as the time from randomization to either the first event of recurrence or death from any cause. Operative methods and pathology results were recorded according to the Japanese classification of gastric carcinoma, second English edition [11]. Hospital mortality was defined as postoperative death of any cause within 30 days, or death within the same hospitalization period as that for the operation. Patients were followed every 3 months until 
five years postoperatively. Adjuvant therapy was not permitted before the recurrence of cancer.

\section{Statistical considerations}

For the non-inferiority design, one-sided log-rank test with a non-inferiority margin was used in order to show statistically that the hazard rate of the non-bursectomy group was no less than that of the bursectomy group. We planned initially to recruit 200 patients, with an $\alpha$ error of 0.1 and statistical power of $80 \%$. This allowed for detecting a hazard ratio with a non-inferiority margin of 1.56 in the non-bursectomy group with the estimation of a $60 \%$ 5-year OS in the bursectomy group. The projected accrual period and follow-up period were 3 and 5 years, respectively. The required sample size was calculated by a simulated-based approach with 100,000 replications to obtain a reasonable size, because a more conventional approach (for example, Freedman's formula) tends to overestimate the sample size as the hazard ratio margin is more greatly separated from one when there is a high censoring rate. After the registration of 204 patients, we amended the sample size and analysis to correct the estimation of 5-year OS in the bursectomy group as $75 \%$ and to reduce the $\alpha$ error. The amended sample size was 464 , with an $\alpha$ error of 0.05 , statistical power of $80 \%$, and a hazard ratio non-inferiority margin of 1.50 , with a 8 -year accrual period (in total) and 5-year follow-up. The hazard ratio margin was designed as corresponding to a non-inferiority margin of $10 \%$ in 5-year OS.

Differences in proportions between the two groups were evaluated using Fisher's exact test or the $\chi^{2}$ test. Differences in continuous variables, including age and tumor size, between the two groups were tested with the MannWhitney $U$-test. Data from all eligible patients were analyzed for OS and RFS on an intention-to-treat basis. Survival curves were estimated by the Kaplan-Meier method and compared using the log-rank test. Hazard ratios were calculated by Cox regression analysis without adjustment for stratification factors. Two-sided $P$ values were used for testing superiority, because our interest in superiority was whether the two groups were different regardless of the direction of the difference, and hence two-sided tests were used as usual. However, one-sided $P$ values were used for testing non-inferiority, because onesided tests were performed following the study design for non-inferiority. All non-inferiority tests were conducted using the handicap log-rank test setting the hazard ratio non-inferiority margin of 1.50. All $P$ values were reported as statistically significant if $P<0.05$, to provide conventional interpretation of results. Statistical analysis was performed using SPSS Statistics software, version 17.0 (SPSS, Chicago, IL, USA) and the $\mathrm{R}$ programming language.
Interim analysis

In January 2007, a large-scale randomized controlled trial evaluating the efficacy of adjuvant S-1 chemotherapy for stage II/III gastric cancer patients reported positive results [12]. Since then, adjuvant S-1 chemotherapy has been the new standard treatment for stage II/III gastric cancer in Japan. As our study did not permit adjuvant treatment, including S-1 chemotherapy, we decided to close accrual of our study in January 2007.

Although the interim analysis was not initially planned, the steering committee of this study proposed interim analyses to examine survival and to provide early release of the results, because the time to definitive analysis ( 5 years) was very long. The data and safety monitoring committee of the Osaka University Clinical Research Group for Gastroenterological Surgery approved the interim analyses with the conditions of Korn's criteria for preliminary data release in randomized clinical trials of non-inferiority [13]. After confirmation of all conditions in Korn's criteria, interim analyses were performed in September 2008 and August 2010. The final analysis of survival data is scheduled for 2012 .

\section{Results}

Between July 2002 and January 2007, 210 patients were randomized to either bursectomy (104 patients) or nonbursectomy (106 patients) (Fig. 1). Patient characteristics were well balanced between the two groups (Table 1). Total gastrectomy was performed for 22 patients $(21.2 \%)$ in the bursectomy group and 27 patients $(25.5 \%)$ in the non-bursectomy group, while 12 bursectomy (11.5\%) and 14 non-bursectomy $(13.2 \%)$ patients underwent splenectomy. Only one $(0.9 \%)$ non-bursectomy patient underwent distal pancreatectomy. The reasons for R1 resection were positive lavage cytology, except in one non-bursectomy patient with a positive proximal margin.

As reported previously [10], bursectomy required a longer operative time, with a median added time of $27 \mathrm{~min}$ in patients with combined resection and $26 \mathrm{~min}$ in patients without combined resection. Intraoperative blood loss was also greater in the bursectomy group (median $475 \mathrm{~mL}$ ) than in the non-bursectomy group (median $350 \mathrm{~mL}$ ) $(P=0.047)$, while other surgical factors did not vary significantly. The overall morbidity rate was $14.3 \%$ for both groups. The median amylase levels in drainage fluid on postoperative day 1 were similar in the two groups $(P=0.543)$. The hospital mortality rate was $0.95 \%$, with one patient death in each group.

At the time of the first interim analysis in September 2008, 3-year OS was $86.4 \%$ for the bursectomy group and 
Fig. 1 Distribution of the patients

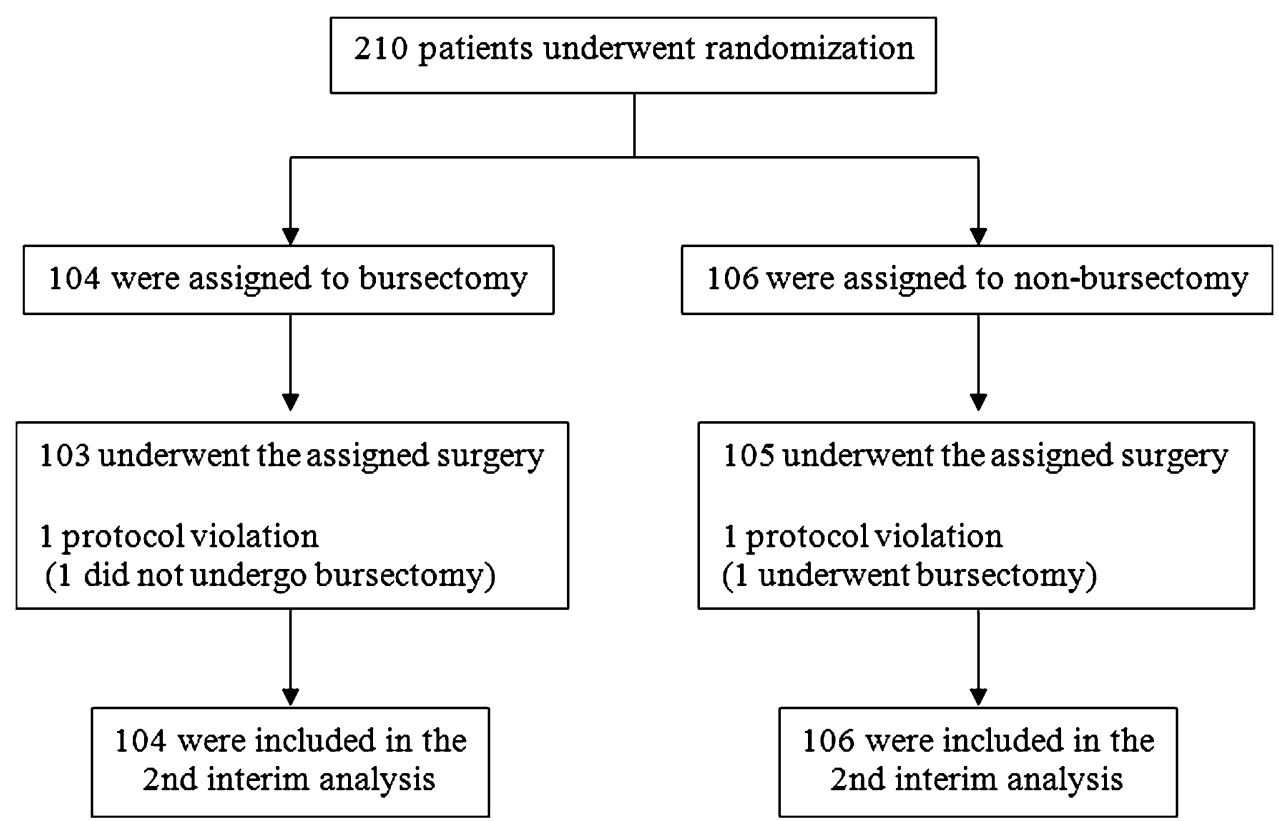

$79.1 \%$ for the non-bursectomy group [14]. The hazard ratio for death in the non-bursectomy group was 1.55 (95\% CI $0.84-2.84 ; P=0.155$ for superiority; $P=0.540$ for noninferiority). By the time of the second interim analysis in August 2010, the median patient follow-up was 46 months; there had been 19 deaths in the bursectomy group and 25 deaths in the non-bursectomy group. The 3-year OS remained better in the bursectomy group $(85.6 \%)$ than in the non-bursectomy group (79.6\%) (Fig. 2). The hazard ratio for death in the non-bursectomy group was $1.44(95 \%$ CI $0.79-2.61 ; P=0.232$ for superiority; $P=0.443$ for non-inferiority).

At the second interim analysis, 24 and 27 recurrences had been recorded in the bursectomy and non-bursectomy groups, respectively. The 3-year RFSs were 77.5 and $75.6 \%$ in the bursectomy and non-bursectomy groups, respectively (Fig. 3). The hazard ratio for recurrence in the non-bursectomy group was 1.18 (95\% CI $0.68-2.04$; $P=0.563$ for superiority; $P=0.192$ for non-inferiority). The most frequent site of first tumor recurrence was the peritoneum, as seen in nine patients in the bursectomy group and 14 patients in the non-bursectomy group (Table 2).

We performed a subgroup analysis examining pathological T stage. Among the 162 serosa-negative (pT1-T2) patients, 3-year OS was 90.5 and $88.1 \%$ for the bursectomy and non-bursectomy groups, respectively (Fig. 4a). In contrast, among the 48 serosa-positive (pT3-T4) patients, 3 -year OS was $69.8 \%$ for the bursectomy patients, in contrast to $50.2 \%$ for the non-bursectomy group (Fig. 4b). The hazard ratios for death in the non-bursectomy group by pathological stage were 1.15 (95\% CI $0.51-2.61$; $P=0.734$ for superiority; $P=0.263$ for non-inferiority) for serosa-negative patients and 2.16 (95\% CI 0.89-5.22; $P=0.081$ for superiority; $P=0.791$ for non-inferiority) for serosa-positive patients. Regarding RFS, serosa-negative patients showed similar results in the two groups ( $P=0.673$ for superiority) (Fig. 5a), while serosa-positive patients showed distinct differences in survival between the two groups ( $P=0.086$ for superiority) (Fig. 5b).

\section{Discussion}

It has been proposed that prophylactic bursectomy prevents peritoneal recurrences by eliminating cancer cells scattered on the lining of the post-gastric cavity; however, the clinical value of bursectomy has not been demonstrated previously. In our randomized controlled trial, experienced surgeons safely performed D2 gastrectomy with bursectomy without increasing major surgical complications, despite longer operative times and increased intraoperative blood loss [10]. The first and second interim analyses revealed that the bursectomy group had better OS than the non-bursectomy group, although these differences were not statistically significant.

The cavity of the bursa omentalis is not a closed chamber, but opens to the abdominal space through the foramen of Winslow. Yamamura et al. [8] reported that carcinoembryonic antigen or cytokeratin 20 mRNA was detected in peritoneal washes from the bursa omentalis, as well as from the Douglas pouch and the left subphrenic cavity. Resection of the bursa omentalis, the most frequent site of peritoneal seeding from the stomach, may eliminate the majority of cancer cells seeded within the peritoneum [15]. In our study, serosa-positive patients, who have the 
Table 1 Patient characteristics

\begin{tabular}{|c|c|c|c|}
\hline & $\begin{array}{l}\text { Bursectomy } \\
(n=104)\end{array}$ & $\begin{array}{l}\text { Non-bursectomy } \\
(n=106)\end{array}$ & $P$ valu \\
\hline \multicolumn{4}{|c|}{ Age (years) } \\
\hline Median & 65 & 63 & \multirow[t]{2}{*}{0.099} \\
\hline Range & $31-79$ & $34-78$ & \\
\hline \multicolumn{4}{|l|}{ Gender } \\
\hline Male & 73 & 77 & \multirow[t]{2}{*}{0.761} \\
\hline Female & 31 & 29 & \\
\hline \multicolumn{4}{|c|}{ Tumor size $(\mathrm{cm})$} \\
\hline Median & 4.3 & 4.5 & \multirow[t]{2}{*}{0.311} \\
\hline Range & $0.9-11.0$ & $1.5-12.0$ & \\
\hline \multicolumn{4}{|c|}{ Clinical $\mathrm{T}$ stage } \\
\hline $\mathrm{cT} 2$ & 61 & 67 & \multirow[t]{2}{*}{0.572} \\
\hline cT3 & 43 & 39 & \\
\hline \multicolumn{4}{|c|}{ Clinical N stage } \\
\hline $\mathrm{cNO}$ & 59 & 61 & \multirow[t]{2}{*}{1.000} \\
\hline $\mathrm{cN} 1$ & 45 & 45 & \\
\hline \multicolumn{4}{|c|}{ Pathological $\mathrm{T}$ stage } \\
\hline pT1 & 17 & 19 & \multirow[t]{3}{*}{0.902} \\
\hline pT2 & 62 & 64 & \\
\hline pT3-4 & 25 & 23 & \\
\hline \multicolumn{4}{|c|}{ Pathological N stage } \\
\hline $\mathrm{pN} 0$ & 49 & 60 & \multirow[t]{3}{*}{0.119} \\
\hline $\mathrm{pN} 1$ & 37 & 24 & \\
\hline $\mathrm{pN} 2-3$ & 18 & 22 & \\
\hline \multicolumn{4}{|c|}{ Residual tumor } \\
\hline R0 & 101 & 102 & \multirow[t]{2}{*}{1.000} \\
\hline $\mathrm{R} 1$ & 3 & 4 & \\
\hline
\end{tabular}

The $P$ values for gender, clinical $\mathrm{T}$ stage, clinical $\mathrm{N}$ stage, and residual tumor were calculated by Fisher's exact test; those for pathological $\mathrm{T}$ stage and pathological $\mathrm{N}$ stage were calculated by the $\chi^{2}$ test; and those for age and tumor size were calculated by the Mann-Whitney $U$-test

highest probability of peritoneal recurrence, displayed differences in 3-year OS of approximately $20 \%$ between the two groups. The bursectomy group showed a decreased frequency of peritoneal recurrence, while nodal recurrence occurred at similar rates in the two groups. The total numbers of dissected lymph nodes were similar in the two groups, with a median of 38 (range 11-98) in the bursectomy group and 37 (range 7-97) in the non-bursectomy group [10]. Even those lymph nodes dissected in the operative field of bursectomy, such as No. 6 (infrapyloric), No.14v (along the superior mesenteric vein), and No.8a (along the common hepatic artery), were similar in the two groups (data not shown). These results suggested that the survival benefit of bursectomy was attributable not to more accurate lymphadenectomy, but to the en-bloc removal of free cancer cells or micrometastases contained in the bursa omentalis.

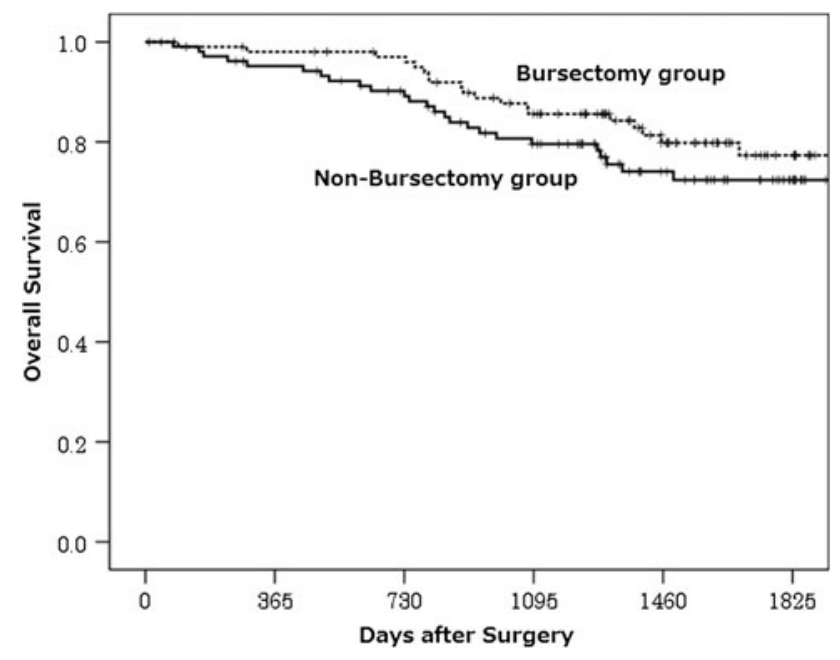

Fig. 2 Overall survival in all patients by treatment group

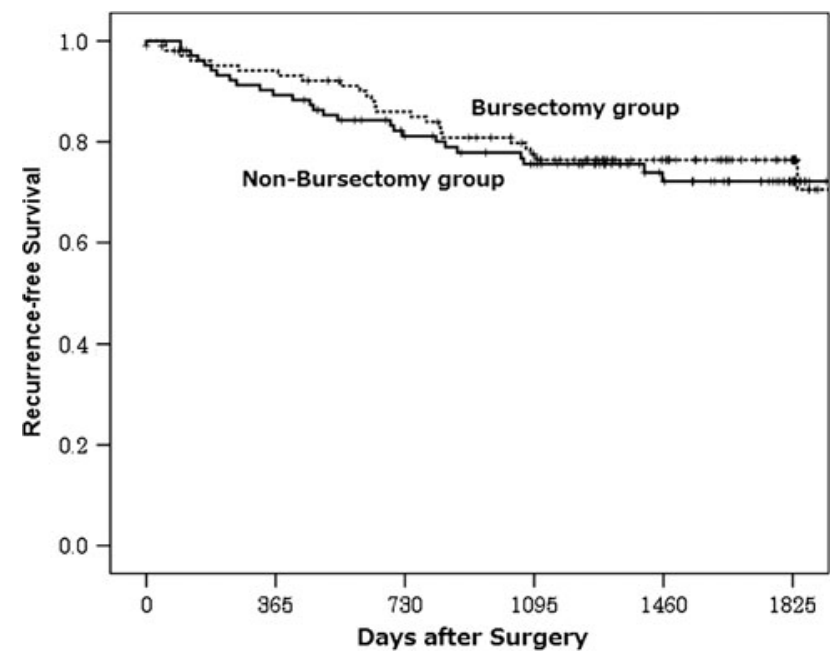

Fig. 3 Recurrence-free survival in all patients by treatment group

As randomized controlled trials preserve type I and II error rates, the interim results of the trial must be powerful for a data-monitoring committee to stop a trial. The public cannot access data from the interim analyses unless the data meet the criteria for early termination of the study. Because non-inferiority trials often require a long followup period for definitive analysis, the early release of the data would be potentially useful to patients who face a treatment decision. Korn et al. [13] suggested that the early release of outcome data could only be done under specific conditions without harming the future conduct of the trial and without being misleading. As the present study satisfied all Korn's conditions, the study steering committee and the data and safety monitoring committee approved the early release of the interim analysis results to the public.

The difference in RFS between the two study groups in our trial was not clear in comparison to that for OS. 
Table 2 Site of first tumor recurrence

\begin{tabular}{lll}
\hline & $\begin{array}{l}\text { Bursectomy } \\
(n=104)\end{array}$ & $\begin{array}{l}\text { Non-bursectomy } \\
(n=106)\end{array}$ \\
\hline Any site of recurrence & 24 & 26 \\
Peritoneum & 9 & 14 \\
Lymph nodes & 7 & 5 \\
Liver & 4 & 6 \\
Others & 4 & 2 \\
\hline
\end{tabular}
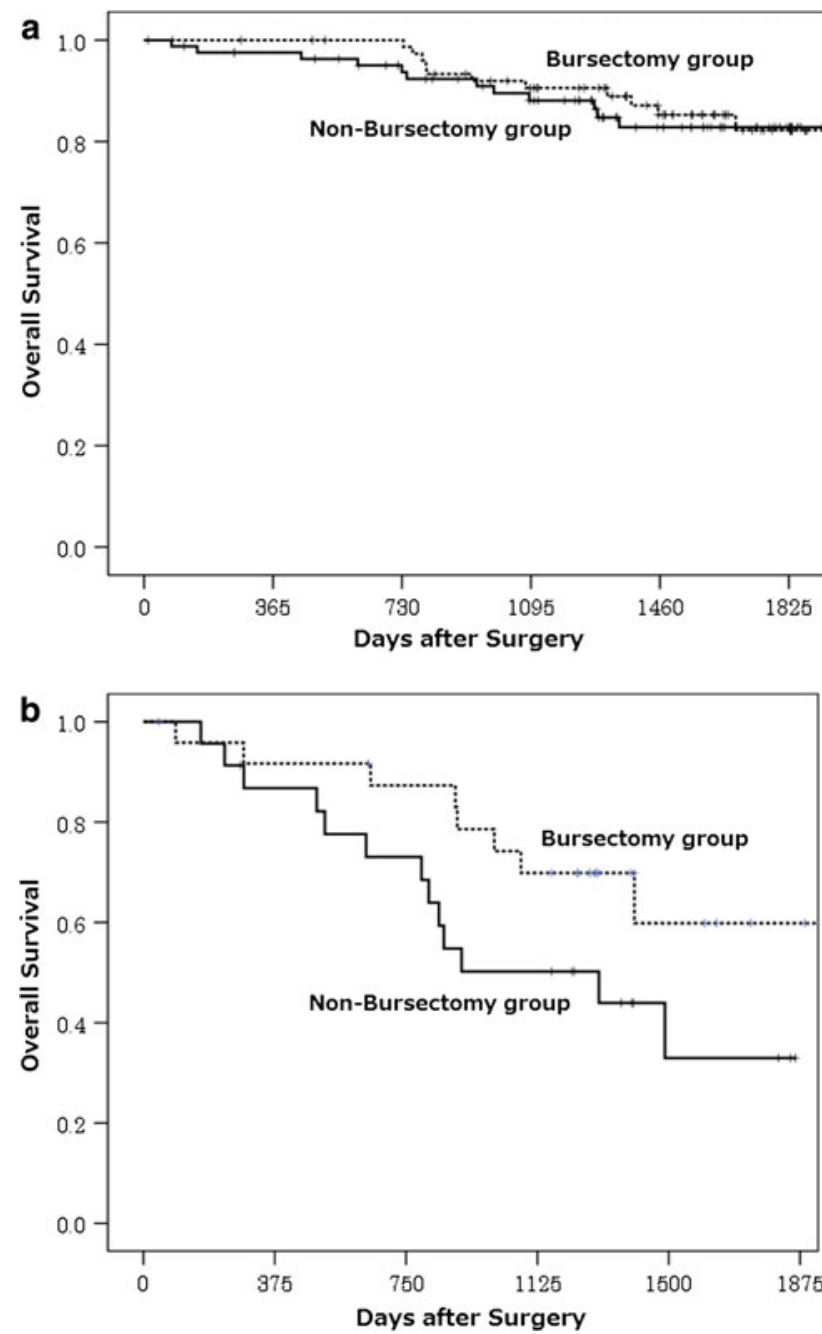

Fig. 4 Overall survival in patients with serosa-negative tumors (a) and those with serosa-positive tumors (b) by treatment group

In clinical trials of gastric cancer treatments, it is difficult to examine peritoneal recurrence, which is the most frequent pattern of relapse. We therefore focused on the endpoint of OS, not on RFS, in the interim analyses. The number of recurrence events (51 events) was smaller than expected, while the number of deaths (44 events) was similar in frequency to that reported in previous studies. The immature results on recurrence for the interim
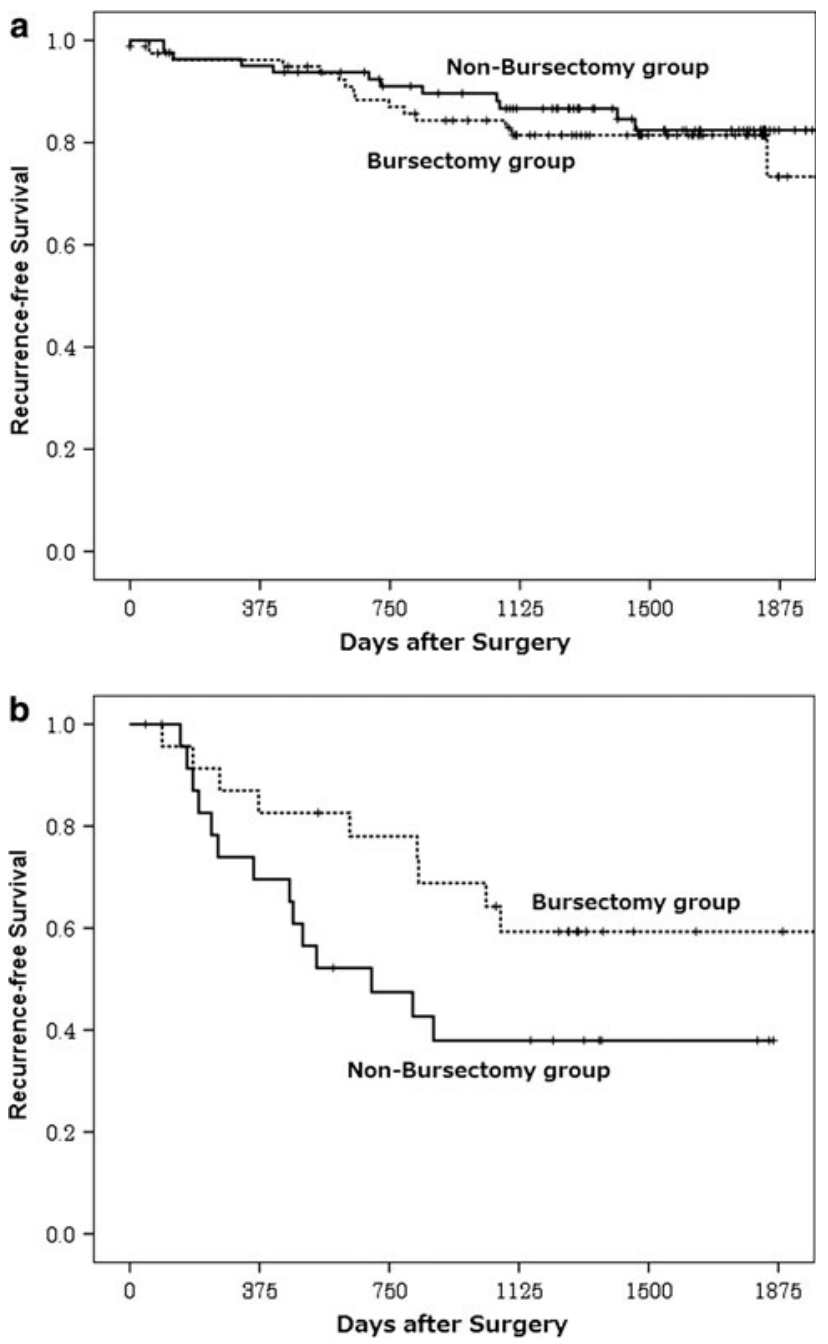

Fig. 5 Recurrence-free survival in patients with serosa-negative tumors (a) and those with serosa-positive tumors (b) by treatment group

analysis may have provided only small differences in RFS between the two groups. More accurate results concerning RFS will be provided in the final analysis.

This study is the first randomized controlled trial to evaluate omental bursectomy in gastric cancer surgery, although it may be under-powered to provide a definitive conclusion. The interim analyses suggest that bursectomy may improve survival in gastric cancer patients and should not be abandoned as a futile procedure until more definitive data can be obtained. The Japan Clinical Oncology Group (JCOG) is now conducting a large-scale randomized controlled trial (JCOG1001) with the recruitment of 1000 patients with cT3-T4 tumors to confirm the superiority of bursectomy in terms of overall survival.

Acknowledgments The authors would like to thank Professor Kunio Okajima for helpful advice; and Dr. Hiroshi Furukawa, Dr. Toshimasa Tsujinaka, Dr. Chikara Ebisui, Dr. Shigeyuki Tamura, 
Dr. Masakatsu Kinuta, and Dr. Norimasa Fukushima for participating in this trial.

\section{References}

1. Jinnai D. Theory and practice of the extended radical operation for gastric cancer. Rinsho Geka. 1967;22:19-24. (in Japanese).

2. Maruyama K, Okabayashi K, Kinoshita T. Progress in gastric cancer surgery in Japan and its limits of radicality. World J Surg. 1987;11:418-25.

3. Groves EWH. On the radical operation for cancer of the pylorus, with especial reference to the advantages of the two-stage operation and to the question of the removal of the associated lymphatics. BMJ. 1910;12:366-70.

4. Oglivie WH. Cancer of the stomach. Surg Gynecol Obstet. 1939; 68:295-305.

5. Hagiwara A, Sawai K, Sakakura C, Shirasu M, Ohgaki M, Yamasaki J, et al. Complete omentectomy and extensive lymphadenectomy with gastrectomy improves the survival of gastric cancer patients with metastases in the adjacent peritoneum. Hepatogastroenterology. 1998;45:1922-9.

6. Japanese Gastric Cancer Association. Gastric cancer treatment guidelines. Tokyo: Kanehara; 2004. (in Japanese).

7. Yoshikawa T, Tsuburaya A, Kobayashi O, Sairenji M, Motohashi $\mathrm{H}$, Hasegawa $\mathrm{S}$, et al. Is bursectomy necessary for patients with gastric cancer invading the serosa? Hepatogastroenterology. 2004;51:1524-6.

8. Yamamura Y, Ito S, Mochizuki Y, Nakanishi H, Tatematsu H, Kodera Y, et al. Distribution of free cancer cells in the abdominal cavity suggests limitation of bursectomy as an essential component of radical surgery for gastric carcinoma. Gastric Cancer. 2007;10:24-8.

9. Fujita J, Tsukahara Y, Ikeda K, Akagi K, Kan K, Hata S, et al. Evaluation of omentum preserving gastrectomy for advanced gastric cancer. Jpn Gastroenterol Surg. 2003;36:1151-8. (in Japanese).

10. Imamura H, Kurokawa Y, Kawada J, Tsujinaka T, Takiguchi S, Fujiwara Y, et al. Influence of bursectomy on operative morbidity and mortality after radical gastrectomy for gastric cancer: results of a randomized controlled trial. World J Surg. 2011;35:625-30.

11. Japanese Gastric Cancer Association. Japanese classification of gastric carcinoma-2nd English edition. Gastric Cancer. 1998;1:10-24.

12. Sakuramoto S, Sasako M, Yamaguchi T, Kinoshita T, Fujii M, Nashimoto A, et al. Adjuvant chemotherapy for gastric cancer with S-1, an oral fluoropyrimidine. N Engl J Med. 2007;357: 1810-20.

13. Korn EL, Hunsberger S, Freidlin B, Smith MA, Abrams JS. Preliminary data release for randomized clinical trials of noninferiority: a new proposal. J Clin Oncol. 2005;23:5831-6.

14. Kurokawa Y, Fujiwara Y, Takiguchi S, Fujita J, Imamura H, Tsujinaka T, et al. Randomized controlled trial of omental bursectomy for resectable cT2-3 gastric cancer. Proc Am Soc Clin Oncol (Gastrointest Cancers Symposium). 2011: \#72 (abstr).

15. Hagiwara A, Takahashi T, Sawaki A, Taniguchi H, Shimotsuma M, Okano S, et al. Milky spots as the implantation site for malignant cells in peritoneal dissemination in mice. Cancer Res. 1993;53:687-92. 